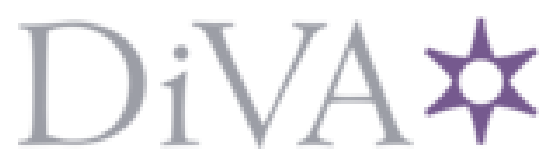

http://www.diva-portal.org

This is the published version of a chapter published in Weighing and Reasoning: A Festschrift for John Broome.

Citation for the original published chapter:

Hattiangadi, A. (2015)

Metasemantics out of Economics?.

In: Andrew Reisner and Iwao Hirose (ed.), Weighing and Reasoning: A Festschrift for John Broome (pp. 52-60). Oxford: Oxford University Press

N.B. When citing this work, cite the original published chapter.

Permanent link to this version:

http://urn.kb.se/resolve?urn=urn:nbn:se:su:diva-139780 


\section{Metasemantics out of Economics?}

University Press Scholarship Online

\section{Oxford Scholarship Online}

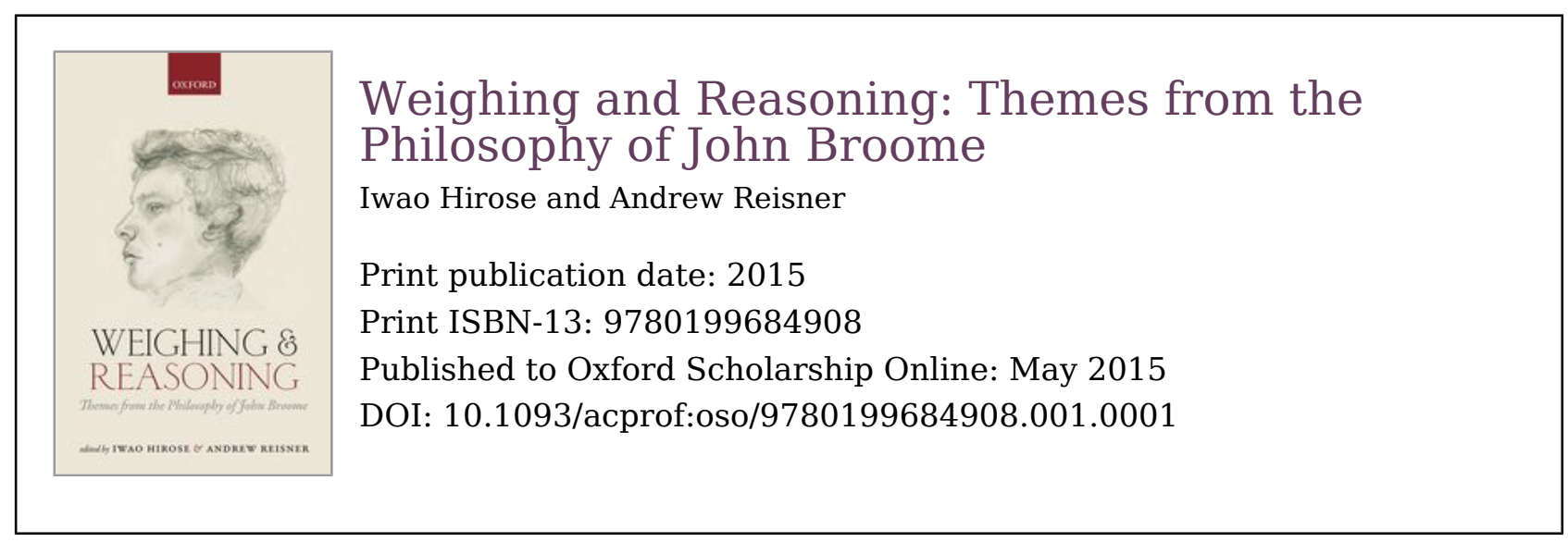

\section{Metasemantics out of Economics?}

Anandi Hattiangadi

DOI:10.1093/acprof:oso/9780199684908.003.0004

\section{Abstract and Keywords}

This chapter discusses the application of formal methods from social choice theory to the metasemantic question of whether radical interpretation is possible. Radical interpretation involves deducing semantic truths from non-semantic truths by appeal to certain a priori principles or criteria, such as the principle of charity. A familiar view is that the intended interpretation is the one that best meets a combination of constraints. It is suggested that this situation can be modelled as follows: each constraint determines a binary relation on the set $X$ of interpretations (' $X$ is at least as good as $y$ with respect to the ith constraint') that is transitive and complete. The radical interpreter's task is to determine an overall ordering as a function from the profile of individual orderings. The application of Arrow's theorem in this context is discussed.

Keywords: radical interpretation, Arrow's theorem, metasemantics, principle of charity, eligibility 
In Ethics out of Economics, John Broome argues for the use of formal economic techniques in philosophical ethics. He says, for instance, 'economists have developed for their own purposes sophisticated methods of analysis that turn out to be useful in philosophical ethics. They can help not only with questions of practical ethics, but also with fundamental issues in ethical theory' (Broome 1999: 1). As Broome points out, the formal aspect of utility theory 'is nothing more than the axioms and the theorems. It can be reinterpreted by substituting in place of preference any other relation that happens to satisfy the axioms' (Broome 1999: 9). Can formal methods of economics be usefully applied to philosophical issues outside of ethics? In this chapter, I shall argue for the use of certain formal techniques from economics in metasemantics.

\section{Radical Interpretation}

Metasemantics is the study of the determinants of meaning, or other semantic properties, such as truth conditions, reference, or truth. One central question of metasemantics is whether radical interpretation is possible. Suppose that Mary is a perfectly rational being who knows $N_{1}$-a statement of all positive non-semantic truths about the actual world, including all of the truths of physics, chemistry, biology, geology, psychology, and so forth. ${ }^{1}$ (It is a positive non-semantic truth that a particular particle, $e$, has a negative charge; it is a negative truth that $e$ is not a ghost.) In addition to the positive truths, suppose that Mary knows T-a 'that's all' statement to the effect that nothing more exists than is needed to satisfy $N_{1}{ }^{2}$ Now, let $S_{1}$ be a statement of all the semantic truths about the actual world, that is to say, truths concerning the distribution of such semantic properties as meaning, truth (p.53) conditions, truth-values, reference, content, and so forth. $S_{1}$ is a massive, global interpretation, a statement of how things actually are in all semantic respects. Suppose that a radical interpreter knows all of the non-semantic truths about the actual world-that is, she knows $N_{1} T$. Suppose, further, that she is perfectly rational and omniscient with regard to all a priori knowable truths. Radical interpretation is possible only if it is possible for such an ideal being to logically 


\section{Metasemantics out of Economics?}

deduce $S_{1}$ from what she knows, namely, $N_{1} T$ together with some a priori truths. ${ }^{3}$ As an ideally rational being, the radical interpreter is constrained by principles of rationality. If radical interpretation is possible, then these principles of rationality, together with the a posteriori information $\left(N_{1}\right)$ and a priori truths must determine the ideal interpreter's choice of $S_{1}$ from the set of interpretations $X=\left\{S_{1} \ldots S_{n}\right\}$, given her knowledge of $N_{1} T{ }^{4}$

Is radical interpretation possible? If it is, then what are the relevant a priori truths governing choice of interpretation? These a priori truths are often characterized as constraints or principles governing the rational selection of interpretations. For instance, one might think that Mary should select the most charitable interpretation in the sense that she should pick the one that maximizes truth in the subject's system of beliefs (Davidson 1990), or which would render the subject maximally rational, given her experiences (Lewis 1974). ${ }^{5}$ Alternatively, one might adopt a principle of (p.54) epistemic charity according to which she should maximize a subject's knowledge (Williamson 2004). Perhaps Mary should select the interpretation that assigns the most 'natural' or 'joint carving' properties as referents of concepts and expressions (Lewis 1999; Sider 2011). According to one version of Lewis's view, the perfectly natural properties are those that are referred to by our most fundamental physical theory, and a property $F$ is more natural than $G$ iff the members of $F$ resemble one another more closely in perfectly natural respects than the members of $G$. Alternatively, one might think that the assignments should best match certain causal relations (Field 1975; Williams 2007). All of these suggestions have some initial plausibility.

The trouble is that the constraints that have been suggested have also been shown to be insufficient-at least when considered in isolation. As is well known, for instance, $S_{1}$ is not the most charitable interpretation. As many philosophers have argued, it is possible to construct a crazy interpretation that is as charitable as $S_{1}$ but is incompatible with it (Quine 1968;

Field 1975; Putnam 1980; Kripke 1982; Williams 2007). The

\footnotetext{
Page 3 of 15
}

PRINTED FROM OXFORD SCHOLARSHIP ONLINE (www.oxfordscholarship.com). (c) Copyright Oxford University Press, 2017. All Rights Reserved. Under the terms of the licence agreement, an individual user may print out a PDF of a single chapter of a monograph in OSO for personal use (for details see http://www.oxfordscholarship.com/page/privacy-policy). Subscriber: 


\section{Metasemantics out of Economics?}

trick is to permute the referents of names in some systematic way and then alter the meanings of predicates so that the changes 'cancel each other out' and, at the level of whole sentences or thoughts, truth-values remain unchanged. This means that Mary, appealing to the principle of alethic charity and knowledge of $N_{1} T$, could not rule out crazy interpretations a priori, and hence could not deduce $S_{1}$ a priori from $N_{1} T$. Similar objections have been raised to the appeal to naturalness (Williams 2007), rationality (Eriksson and Háyek 2007), and causal constraints (Kripke 1982; Lewis 1999).

A natural reaction to these concerns is to consider whether a number of constraints might work in concert. Indeed, this thought may well have been in play all along-in 'Radical Interpretation', Lewis puts forward no less than six constraints, and suggests that they work together. Even if one or more constraints is emphasized (such as naturalness in Lewis (1999)), there is usually a compositionality constraint assumed in the background (cf. Field 1975). Sider (2011), in a recent defence of a broadly Lewisian approach to metasemantics suggests that 'joint-carvingness' (much like Lewisian naturalness) can be combined with other constraints to determine the intended interpretation. The thought is that Mary is rationally required to select the interpretation that is best overall in meeting a number of individual constraints.

Here is where economics comes in. Let's say that $X$ is the set of alternative interpretations and that each constraint determines a weak ordering of interpretations denoted $x R_{i} y$ (' $x$ is at least as good as $y$ with respect to the ith constraint'). From $R_{i}$, a strict ordering $P_{i}$ (' $x$ is better than $y$ with respect to the ith constraint') and indifference $I_{i}$ (' $x$ is equally as good as $y$ with respect to the ith constraint') can be defined. For instance, the alethic charity constraint will determine a weak ordering of interpretations, where interpretation $x$ is at least as good as $y$ with respect to alethic charity iff the number of beliefs $x$ renders true is greater than or equal to the number of beliefs $y$ renders true. Similarly, the naturalness constraint will determine a weak (p.55) ordering of interpretations such that $x$ is at least as good as $y$ with respect to naturalness iff the

\footnotetext{
Page 4 of 15
} 


\section{Metasemantics out of Economics?}

naturalness of assignments of referents to lexical items made by $x$ is greater than or equal to the naturalness of assignments of referents to lexical items made by $y$. And so on, for each constraint. A profile is the $n$-tuple of individual orderings $\left\{R_{i}\right\}$.

What does Mary do with this profile? She needs to find a way of aggregating the individual orderings of interpretations so as to determine which interpretation is best overall. She needs to arrive at an overall ordering, $x R y$, which is a function of the individual orderings-call that function an OIF for 'overall interpretation function'. Of course, there are countless OIFs. Some rank $S_{1}$ as best overall; others rank some crazy interpretations highest. Which one to use? Once again, Mary needs some kind of rational basis for picking one such function over the rest. Her predicament is structurally analogous to those investigated by social choice theory. ${ }^{6}$

\section{Social Choice Theory}

Social choice theorists investigate functions from individual preferences $\left(R_{i}\right)$ to a social preference $(R)$ over the set of states of the world in the set $X$. Suppose five committee members wish to elect a chair from their ranks. Each member ranks the five candidates according to her own preferences. Each member's preferences determine a weak ordering of states of the world, denoted $x R_{i} y$ ( $x$ is at least as good as $y$ according to the ith member), and from this, a strict ordering $x P_{i} y$ and indifference, $x I_{i} y$ can be defined. The social choice theorist investigates ways to determine an overall, or general preference ordering, $x R y$ from the individual ones. Once again, there are many social welfare functions (SWFs) from individual to overall orderings. The social choice theorist attempts to find constraints that will rule out unreasonable methods of aggregation yielding only the set of aggregation methods that are reasonable (Sen 1999).

Famously, Kenneth Arrow proved that no social welfare function (SWF) could satisfy a small number of seemingly plausible and relatively weak constraints on a SWF (Arrow 1963). First, Arrow assumes that the individual orderings $x R_{i} y$ are transitive (if $x R_{i} y$ and $y R_{i} z$, then $x R_{i} z$ ) and complete-they

\footnotetext{
Page 5 of 15
} 


\section{Metasemantics out of Economics?}

have preferences over all of the alternatives in the set. The additional constraints are as follows:

$\mathbf{U}$ (unrestricted domain):

The domain of the SWF is the set of all logically possible profiles.

(p.56) $\mathbf{P}$ (weak Pareto):

If every individual strictly prefers $x$ to $y$, then $x$ is socially preferred to $y$.

D (non-dictatorship):

There is no individual who dictates the social preference in the sense that if she strictly prefers $x$ over $y$, then $x$ is socially preferred to $y$.

I (independence of irrelevant alternatives):

The social preference between $x$ and $y$ can only depend on individuals' preferences between $x$ and $y$.

Arrow's theorem is that, so long as there are at least three alternative states and a finite number of voters, $\mathbf{U}, \mathbf{P}, \mathbf{D}$, and $\mathbf{I}$ cannot be satisfied (Arrow 1963; Sen 1986).

As Broome suggests, formal economic theories are nothing more than the axioms and the theorems. We can reinterpret them by substituting in place of voter's preferences, different relations that satisfy the axioms. In the next section, I will make a prima facie case for applying Arrow's axioms in the case of radical interpretation, where each individual preference ordering is reinterpreted as an ordering of interpretations based on individual criteria (such as charity, naturalness, rationality, and so forth). Metaphorically, we can imagine the radical interpreter as a committee composed of single-minded individuals ranking interpretations: Charity, who cares only about charity, Natalie, who only cares about naturalness, Radha, who only cares about rationality, and so forth. But that is really just picturesque. It is sufficient that the

\footnotetext{
Page 6 of 15
}

PRINTED FROM OXFORD SCHOLARSHIP ONLINE (www.oxfordscholarship.com). (c) Copyright Oxford University Press, 2017. All Rights Reserved. Under the terms of the licence agreement, an individual user may print out a PDF of a single chapter of a monograph in OSO for personal use (for details see http://www.oxfordscholarship.com/page/privacy-policy). Subscriber: 
axioms are satisfied. I will argue in the next section that they are.

\section{Back to Radical Interpretation}

Arrow assumes that individual preference orderings are transitive and complete. Though there have been reasons to question whether these should be weakened in the case of social choice (Sen 1986), there seems to be no reason to weaken these constraints here. Transitivity seems to be analytic of 'at least as good as', 'strictly better than', and 'indifferent between', and the radical interpreter aims to select the interpretation that is best (i.e. strictly better than the rest) overall. Completeness, once again, might be implausible if the individual orderings are meant to capture the preferences of individuals over infinitely many states of the world, given human cognitive limitations. However, no comparable cognitive limitations plague the radical interpreter, who can work out each individual ordering a priori, given full information about any logically possible $N_{i}$ together with full information about every logically possible $S_{i}$.

$\mathbf{U}$ says that the domain of the SWF is the set of all logically possible profiles. Is it plausible that the domain of the OIF is the set of all logically possible profiles? Initially, this may seem implausible. After all, the radical interpreter is given the nonsemantic truths about the actual world, $N_{1}$, and these, together with a certain criterion, determine an individual ordering. However, this initial appearance is misleading. The reason is that though we are primarily interested in the actual (p.57) world, there are many logically possible worlds with different logically possible $N_{i}$. Many non-semantic truths are contingent, and if they had been different, the criteria would determine different individual orderings of interpretations. For instance, consider a world, $w$, that differs from our world in non-semantic facts about our use of 'cat' and 'dog', and where 'cat' means 'dog' and vice-versa. If we let $N_{2}$ be a statement of all non-semantic truths at $w$, and let $S_{2}$ be a statement of the semantic truths at $w$, the OIF should rank $S_{2}$ over $S_{1}$ in relation to $w$, but $S_{1}$ over $S_{2}$ in relation to the actual world. 


\section{Metasemantics out of Economics?}

Of course, one might think that all we need is an OIF that works for the actual world. Who cares whether it gets the right results in all logically possible worlds? However, in radical interpretation, the principles governing aggregation must be a priori, and hence, should not depend on knowledge of any contingent a posteriori truths apart from what is contained in $N_{i}$. This suggests, then, that the domain of the OIF ought to be any profile determined by the set $\left\{N_{i} T\right\}$, for all logically possible $N_{i}$.

A further worry one might have is that some criteria are not independent of one another. For instance, alethic charity orders interpretations by how many true assertions and beliefs are assigned to a subject, and epistemic charity orders them by how much knowledge is assigned. Since knowledge entails true belief, the two orderings cannot vary independently of one another. However, Arrow's theorem only requires that there are at least three logically independent voters. There are arguably at least three logically independent criteria-such as, for instance, alethic charity, naturalness, and causality.

$\mathbf{P}$ says that if interpretation $x$ is better than $y$ according to each individual criterion, then $x$ is better than $y$ overall. This seems plausible. The overall ranking of the interpretations should be wholly determined by the individual rankings in relation to the criteria. For, there is nothing else that could be relevant to determining the overall ranking, since all of the relevant truths, both a priori and a posteriori, have been taken into consideration in the determination of the profile. If the overall ranking were not determined by individual rankings alone, then something else (either a falsehood or a randomizing mechanism) would play a role in determining the overall ranking. But this would tell against the overall ranking having a fully rational basis.

D says that no individual dictates the social preference. This is clearly plausible in the case of social choice, since we want to make sure that no single voter's ranking determines the overall ranking. In the metasemantic case, $\mathbf{D}^{*}$ says that there is no single criterion whose strict ranking dictates the overall ranking. That is, there is no $P_{i}$ which is such that for all $x, y$, if

\footnotetext{
Page 8 of 15
}

PRINTED FROM OXFORD SCHOLARSHIP ONLINE (www.oxfordscholarship.com). (c) Copyright Oxford University Press, 2017. All Rights Reserved. Under the terms of the licence agreement, an individual user may print out a PDF of a single chapter of a monograph in OSO for personal use (for details see http://www.oxfordscholarship.com/page/privacy-policy). Subscriber: 


\section{Metasemantics out of Economics?}

$x P_{i} y$, then $x P y$. Is this plausible in this case? It seems to me that it is, though for very different reasons.

In the case of radical interpretation, if any individual constraint determined the overall ranking, the wrong result would be determined. This is because each individual constraint, considered entirely in isolation, does not rank the intended interpretation highest. For instance, alethic charity ranks many interpretations higher than $S_{1}$. For, given human fallibility, there are many sentences that we assent to, or hold true, (p.58) which are false. Now, consider an interpretation, $S_{3}$, which assigns meanings in such a way that all of our true beliefs come out true, and in addition, some of our false beliefs come out true. For instance, suppose that some subject actually believes that the Earth is flat. Suppose that $S_{3}$ assigns the Earth to the extension of 'flat' in the subject's language, so that 'the Earth is flat' comes out true. The meaning of 'flat' may thereby become gruesome (like Goodman's predicate, 'grue'), referring to all the flat things and only the flat things with the exception of the Earth. Gruesome though it may be, $S_{3}$ would be strictly more charitable than $S_{1}$. If the strict ordering in respect of charity were to dictate the overall ordering, then it would be determined that $S_{3}$ is true, rather than $S_{1}$. That $S_{3}$ might wind up being wildly unnatural or noncompositional is beside the present point, because compositionality and naturalness are further constraints, and we are considering an ordering with respect to alethic charity alone.

Similarly, consider an interpretation, $S_{4}$, that maps every name onto a microphysical particle, and every predicate onto a highly natural property. ${ }^{7} S_{4}$ will be strictly more natural than $S_{1}$. For we speak of many highly unnatural things: of shoes and ships and sealing wax, of cabbages and kings. Once again, $S_{4}$ might well be less rationalizing, or involve fewer causal relations than $S_{1}$, but that is neither here nor there, since we are considering a strict ordering by naturalness alone. Similar points can be raised against the epistemic constraint, since we are far from omniscient, against the causal constraint, because sometimes we talk about things that lack causal powers, and so forth. ${ }^{8}$

\footnotetext{
Page 9 of 15
}

PRINTED FROM OXFORD SCHOLARSHIP ONLINE (www.oxfordscholarship.com). (c) Copyright Oxford University Press, 2017. All Rights Reserved. Under the terms of the licence agreement, an individual user may print out a PDF of a single chapter of a monograph in OSO for personal use (for details see http://www.oxfordscholarship.com/page/privacy-policy). Subscriber: 


\section{Metasemantics out of Economics?}

Of course, whether every criterion ranks some interpretation above the intended interpretation depends on what, more precisely, the criteria are. Yet I have left this open to some degree. However, if there were a single criterion that ranked the intended interpretation highest, there would be no need to aggregate-that criterion would be all that the radical interpreter would need to deduce $S_{1}$ from $N_{1}$. Indeed, if there were such a constraint, the radical interpreter would be illadvised to aggregate. But then, what of the intuitive plausibility of various forms of charity, naturalness, causality, and so forth? Those considerations would then turn out to be irrelevant to the determination of meaning. Thus, it seems that we cannot let the strict ordering determined by any individual criterion dictate the strict ordering overall, irrespective of the orderings with respect to other criteria. There should be no metasemantic dictators. ${ }^{9}$

(p.59) I says that the overall ranking of interpretations $x$ and $y$ can only depend on the rankings of $x$ and $y$ by the individual criteria. This was intuitively plausible in the case of an election-the social ordering of Smith and Jones should depend on the individual orderings of Smith and Jones, and not on how some third candidate, Brown, is ranked. The same seems to hold in the metasemantic case. In this case, the individual orderings of interpretations $x$ and $y$ are determined by the relevant a priori and a posteriori truths. Since the overall ordering should depend on these truths alone, no further truth, such as how some third interpretation $S_{k}$ is ranked, should be relevant to fixing the overall ranking. If the ranking of $S_{k}$ did influence the overall ranking of $S_{i}$ and $S_{j}$, the overall ranking would not have a basis in the a priori and a posteriori truths determining the individual rankings of $S_{i}$ and $S_{j}$.

\section{Concluding Remarks}

I have made a preliminary case for the applicability of Arrovian axioms to radical interpretation. If Arrow's axioms apply, then radical interpretation is impossible, for there is no rational basis for aggregating the orderings determined by individual criteria (and no individual criterion determines the intended interpretation, on its own).

Page 10 of 15

PRINTED FROM OXFORD SCHOLARSHIP ONLINE (www.oxfordscholarship.com). (c) Copyright Oxford University Press, 2017. All Rights Reserved. Under the terms of the licence agreement, an individual user may print out a PDF of a single chapter of a monograph in OSO for personal use (for details see http://www.oxfordscholarship.com/page/privacy-policy). Subscriber: 


\section{Metasemantics out of Economics?}

That said, there are several ways out of Arrow's impossibility theorem, and a fuller discussion would consider whether any such exit strategies are available in the metasemantic case. I have already suggested that rejecting or weakening the axioms seems to be unwarranted in the metasemantic case. Another way out of Arrow's impossibility involves increasing the information contained in the individual rankings. ${ }^{10}$ Crucially, Arrow does not allow for interpersonal comparisons of utility. Yet, if interpersonal comparisons of utility are possible, then versions of Arrow's constraints can be satisfied (Sen 1986). A further question, therefore, is whether intercriterial comparisons are possible.

Though many such further questions remain, I hope to have at least shown that some formal techniques from economics can help to provide a way of addressing an issue in metasemantics: whether radical interpretation is possible.

(p.60) References

Bibliography references:

Arrow, K. (1963) Social Choice and Individual Values. 2nd edn. New York: Wiley.

Broome, J. (1999) Ethics out of Economics. Cambridge:

Cambridge University Press.

Davidson, D. (1990) Radical interpretation. In Inquiries into

Truth and Interpretation. Oxford: Oxford University Press.

Eriksson, L., and Hájek, A. (2007) What are degrees of belief?

Studia Logica 86(2): 183-213.

Field, H. (1975) Noûs 9(4): 375-405.

Hawthorne, J. (2007) Craziness and metasemantics. The Philosophical Review 116 (3): 427-40.

Kripke, S. (1982) Wittgenstein on Rules and Private Language: An Elementary Exposition, Cambridge, Mass.: Harvard University Press.

Lewis, D. (1974) Radical interpretation. Synthese 23: 331-44.

Page 11 of 15

PRINTED FROM OXFORD SCHOLARSHIP ONLINE (www.oxfordscholarship.com). (c) Copyright Oxford University Press, 2017. All Rights Reserved. Under the terms of the licence agreement, an individual user may print out a PDF of a single chapter of a monograph in OSO for personal use (for details see http://www.oxfordscholarship.com/page/privacy-policy). Subscriber: 


\section{Metasemantics out of Economics?}

Lewis, D. (1999) New work on a theory of universals. In Papers in Metaphysics and Epistemology. Cambridge: Cambridge University Press.

Lewis, D. (2008) Languages and language. Reprinted in A. P. Martinich, ed. The Philosophy of Language. Oxford: Oxford University Press.

Morreau, M. (2010) It simply does not add up: Trouble with overall similarity. Journal of Philosophy 107(9): 469-90.

Okasha, S. (2011) Theory choice and Social Choice: Kuhn versus Arrow. Mind 120(477): 83-115.

Putnam, H. (1980) Models and reality. The Journal of Symbolic Logic 45(3): 464-82.

Quine, W. V. (1968) Ontological relativity. The Journal of Philosophy 65(7): 185-212.

Sen, A. (1986) Social choice theory. In K. J. Arrow and M. D. Intriligator (eds.) Handbook of Mathematical Economics, III. London: Elsevier.

Sen, A. (1999) The possibility of social choice. The American Economic Review 89(3): 349-78.

Sider, T. (2011) Writing the Book of the World. Oxford: Oxford University Press.

Williams, J. R. G. (2007) Eligibility and inscrutability. The Philosophical Review 116(3): 361-99.

Williamson, T. (2004) Philosophical 'intuitions' and scepticism about judgment. Dialectica 58(1): 109-53.

Notes:

( $\left.{ }^{1}\right) N$ can include phenomenal truths about the world, such as that a subject has an experience with a particular phenomenal character at a certain time, as well as non-semantic indexical truths, fixing the location of a centre in a world. 


\section{Metasemantics out of Economics?}

${ }^{2}$ ) Any world that satisfies $N_{1} T$ is a minimal non-semantic duplicate of our world. From $N_{1} T$, Mary can infer that there are no ghosts, but not from $N_{1}$ alone.

$\left({ }^{3}\right)$ As I characterize it, radical interpretation is fully reductive, in the sense that the radical interpreter has access to no semantic information whatsoever. This is not always assumed. For instance, Davidson (1990) allows the radical interpreter to have information about what a subject 'holds true', that is, what sentences a subject believes to be true. In one version of Lewis's interpretationism, discussed by Williams (2007), the sentential data includes the sentences that constitute a subject's 'global theory', which naturally suggests that they are sentences that the subject assents to, or is disposed to assent to. But of course such truths are also semantic.

$\left({ }^{4}\right)$ I have simplified somewhat in assuming that the radical interpreter must pick a unique interpretation. Some philosophers, such as Lewis, might be inclined to allow the radical interpreter to select a set of interpretations, to allow for vagueness. Suppose $S_{1}$ assigns a certain precise extension to the English predicate 'bald', and $S_{2}$ assigns an extension that is very slightly different from $S_{1}$ (e.g. it includes people with one more hair on their heads than are included in the extension of 'bald' assigned by $S_{1}$ ). If vagueness is a semantic phenomenon, then arguably non-semantic facts will be indeterminate between $S_{1}$ and $S_{2}$ (Lewis 1974). In other words, the radical interpreter need not be required to select a unique interpretation. However, the simplifying assumption that the radical interpreter should select a unique interpretation is acceptable because nothing in the argument turns on vagueness. Moreover, one could simply allow interpretations to capture semantic vagueness. For instance, $S_{1}$ need not assign a precise extension to 'bald'; it could assign a set of admissible extensions and state that ' $x$ is bald' is determinately true iff $x$ is a member of every extension in the set of admissible interpretations, or something to that effect. If all the truths about vagueness are included in interpretations, then the radical interpreter can once again be expected to select a unique interpretation.

Page 13 of 15

PRINTED FROM OXFORD SCHOLARSHIP ONLINE (www.oxfordscholarship.com). (c) Copyright Oxford University Press, 2017. All Rights Reserved. Under the terms of the licence agreement, an individual user may print out a PDF of a single chapter of a monograph in OSO for personal use (for details see http://www.oxfordscholarship.com/page/privacy-policy). Subscriber: 


\section{Metasemantics out of Economics?}

$\left({ }^{5}\right)$ Strictly speaking, assignments of meaning will be determined more precisely by way of convention. For instance, Lewis argues that 'the convention whereby a population $P$ uses a language $€$ is a convention of truthfulness and trust in $E^{\prime}$ (2008: 658). This is not an independent, fully reductive criterion, however, since Lewis defines these conventions in terms of subjects' preferences and beliefs:

To be truthful in $€$ is to act in a certain way: to try never to utter any sentence of $€$ that are not true in $€$. Thus it is to avoid uttering any sentence of $£$ unless one believes it to be true in $€$. To be trusting in $€$ is to form beliefs in a certain way: to impute truthfulness in $£$ to others, and thus to tend to respond to another's utterance of any sentence of $€$ by coming to believe that the uttered sentence is true in $€$.

This, in effect, constrains what is involved in rationalizing a subject's linguistic behaviour.

$\left({ }^{6}\right)$ Social choice theory, and in particular, Arrow's theorem, has recently been applied to other domains outside of ethics. Michael Morreau (2010) has, for instance, applied Arrow's theorem to similarity judgements, particularly in relation to counterpart theory (' $x$ resembles me at least as much as $y$ overall'). Samir Okasha (2011) has applied them to theory choice in the sciences, where values such as simplicity, scope, fruitfulness (etc.) need to be aggregated into an overall ranking.

( $\left.{ }^{7}\right)$ See Hawthorne (2008) for examples.

$\left({ }^{8}\right)$ One might well worry that this motivation for $\mathbf{D}$ is not a priori-i.e. it depends on our knowledge of semantic truths about the actual world. But this need not jeopardize the project. For we can allow that the principles that govern radical interpretation have something to do with our concepts (e.g. the concept of a language, of a person, of communication, etc.). We can thus 'work back' from our knowledge of semantic truths to uncover the rational principles governing radical interpretation. 


\section{Metasemantics out of Economics?}

$\left({ }^{9}\right)$ Note that $\mathbf{D}$ does not rule out various decision procedures which involve a criterion acting as a tie-breaker. Suppose, for instance, that we start by ranking interpretations by alethic charity and then break ties between the highest ranked by appeal to naturalness. This method would not violate $\mathbf{D}$ since neither alethic charity nor naturalness would alone determine the overall ordering. For suppose that there is a set of interpretations that is tied for first place by charity. There could still be an interpretation that is ranked below those in that set by charity, yet higher than any in that set by naturalness. If naturalness only works as a tie-breaker, the overall ordering will not in this case be determined by either the individual ordering by charity, or by the individual ordering by naturalness alone.

$\left({ }^{10}\right)$ Some suggest that it might be enough to avoid Arrow's impossibility that the individual rankings are cardinal rather than merely ordinal, as Arrow assumes (see Samir Okasha 2011). However, Sen (1986) points out that this is insufficient to avoid the impossibility theorem.

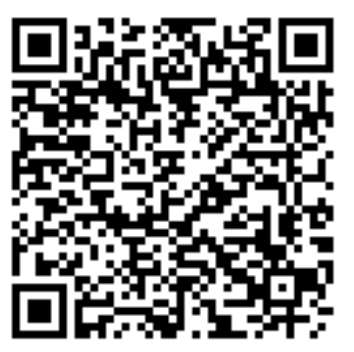

\section{Access brought to you by: Stockholm University}

